\title{
Revisión sistemática de literatura: explotación de información y tecnologías GIS aplicadas para hallar patrones delictivos
}

\author{
Systematic review of literature: exploitation of information \\ and GIS technologies applied to identify criminal patterns
}

\author{
Lorena E. Flores \\ https://orcid.org/0000-0002-1835-1357 \\ lorenaelizabeth.flores@gmail.com \\ Sonia I. Mariño² \\ https://orcid.org/0000-0003-3529-7003 \\ simarinio@yahoo.com
}

ISSN 2071-8748

E-ISSN 2218-3345

Recibido 30 de enero 2019

(c)(1)(0)

Aprobado: 3 de mayo 2019

\section{BY NC SA}

URI: http://hdl.handle.net/11298/972

DOI: https://doi.org/10.5377/entorno.v0i67.7489

\section{Resumen}

La revisión sistemática de la literatura (RSL) es un artículo que sintetiza la evidencia disponible en torno a ciertas preguntas de investigación, aborda aspectos cuantitativos y cualitativos de estudios primarios con el objetivo de resumir la información existente respecto de un tema en particular. Se presenta una revisión sistemática de la literatura acerca de la integración de procesos de explotación de información con tecnologías GIS, y se evalúa su aplicación para el hallazgo de patrones delictivos.

\section{Abstract}

The systematic review of literature (SLR) is an article which synthesizes the available evidence around certain research questions, addresses quantitative and qualitative aspects of primary studies with the purpose of summarizing existing information on a particular topic. A systematic review of the literature on the integration of information exploitation processes with GIS technologies is presented, and it evaluates their application for the discovery of criminal patterns. The construction of this

1 Maestrando de la Maestría en Tecnologías de la Información, Universidad Nacional del Nordeste, Corrientes, Argentina

2 Departamento de Informática. Facultad de Ciencias Exactas y Naturales y Agrimensura. 9 de julio de 1449. CP: 3400 . Corrientes, Argentina, Universidad Nacional del Nordeste 
La construcción de esta RSL se basa en el método propuesto por Kitchenham. Los resultados se exponen considerando las tres etapas del método en donde se explican los estudios primarios seleccionados en relación con las preguntas de investigación planteadas que guían el estudio. Los hallazgos evidencian la escasa aplicación de la integración de ambas tecnologías como estrategia de disminución de riesgos delictivos.

\section{Palabras clave}

Revisión sistemática de la literatura, explotación de la información, minería de datos, GIS, procesos criminales.
SRL is based on the method proposed by Kitchenham. The results are presented by considering the three stages of the method in which the selected primary studies are explained in relation to the research questions that guide the study. The findings show the scarce application of the integration of both technologies as a strategy to reduce criminal risks.

\section{Keywords}

Systematic literature review, information exploitation, data mining, GIS, criminal proceedings.

\section{Introducción}

Una revisión sistemática de la literatura (RSL) es un artículo de síntesis de la evidencia disponible sobre un objeto de estudio determinado. Aborda un análisis de aspectos cuantitativos y cualitativos de estudios primarios con la finalidad de sistematizar y resumir la información relativa a un tema de interés.

Una RSL permite evaluar e interpretar la investigación disponible relevante respecto de un interrogante de investigación particular, en un área temática o fenómeno de interés (Kitchenham, 2004). En Gisbert, J. \& Bonfill (2004), se define a las RSL como "investigaciones científicas en sí mismas, con métodos prefigurados y un ensamblaje de los estudios originales, que sintetizan los resultados de estos". En Benet Rodríguez et al. (2015), se denota como estudios primarios a los estudios individuales que contribuyen a una revisión sistemática, siendo esta última considerada como un estudio secundario.

Este método de indagación incrementa en su relevancia y presencia actualmente. Esto se debe a que estos estudios minuciosos permiten identificar el estado del arte sobre una determinada cuestión, delineando el abordaje en proyectos de investigación, desarrollo e innovación. La construcción de la RSL que se expone, se basa en el método propuesto por Kitchenham (2004).

En este artículo, se presenta una RSL sobre la integración de procesos de explotación de información con tecnologías
GIS y su aplicación para el hallazgo de patrones delictivos. El objetivo es generar información valiosa que oriente en la definición de un mapa del delito.

\section{Explotación de la información}

Para situar el estudio, se definen los conceptos de explotación de información, desde la minería de datos (data mining o MD) y del Descubrimiento de conocimiento en bases de datos (Knowledge Discovery in Databases o KDD).

La explotación de información constituye la subdisciplina de la informática que aporta a la inteligencia de negocios las herramientas para la transformación de información en conocimiento (García Martínez et al., 2011). Un proceso de explotación de información se define como un grupo de tareas relacionadas lógicamente (Britos, 2008) que, a partir de una masa de información con un grado de valor para la organización, se ejecutan para lograr piezas de conocimiento sobre el funcionamiento de algún aspecto de esta, con un grado de valor mayor que la información original (Ferreira et al., 2005; Hann et al., 2007).

Para lograr este objetivo se utilizan las técnicas de minería de datos. Frawley et al. (1992) definen la minería de datos como el proceso mediante el cual se extrae conocimiento comprensible y útil que previamente era desconocido desde bases de datos, en diversos formatos, de manera automática.

La minería de datos es un elemento fundamental de un proceso más amplio que tiene como objetivo el 
descubrimiento de conocimiento en grandes bases de datos o KDD (Fayyad et al., 1996).

Un proceso de explotación de información utiliza las técnicas de minería de datos para el descubrimiento de conocimiento en grandes bases de datos, como se menciona en García Martínez et al. (2011) y Britos (2008).

\section{Tecnologías GIS}

La computación abrió nuevas y potenciales formas de comunicación. Numerosas decisiones dependen de los detalles del entorno inmediato y requieren información sobre lugares específicos en la superficie de la tierra. La información geográfica permite aplicar principios generales a las condiciones específicas de cada ubicación, rastrear lo que sucede en cualquier lugar, y ayuda a entender cómo un lugar difiere de otro. La información geográfica también es esencial para la planificación efectiva y la toma de decisiones en la sociedad moderna (Bernhardsen, 2002).

Los GIS pueden manejar, analizar y representar una amplia variedad de tipo de datos geográficos, generalmente de diversas fuentes. Las bases de datos GIS son utilizadas para organizar y procesar capas de datos geoespaciales (Merry, 2008), y contienen información geográfica valiosa vinculada con un determinado territorio de interés. Desde esta visión, pueden aplicarse en la búsqueda de patrones y regularidades significativas utilizando ingeniería de explotación de información (García Martínez et al., 2012). Existen innumerables aplicaciones de esta tecnología a vastos dominios de conocimiento.

\section{Un dominio de aplicación, los patrones delictivos}

La tecnología GIS se utiliza en el tratamiento de la información de diversos dominios, siendo uno de ellos el referente a la delincuencia. En El-Aziz et al. (2012), se exponen herramientas para el mapeo efectivo del crimen, análisis y la gestión. En este sentido, se coincide con lo presentado en Tillekens et al. (2016), dado que actualmente las evidencias digitales para la investigación del crimen en su mayoría incluyen un componente geoespacial. En Perversi et al. (2007) y Chen et al. (2004) se reportan análisis de incidentes vinculados con la criminalidad.
Una de las medidas para combatir la delincuencia en la Argentina, el Ministerio de Justicia y Derechos Humanos creó el Sistema de Alerta Temprana (SAT) (Perversi et al., 2007). En la actualidad, la información proveniente del SAT se trata mediante el análisis estadístico, sin aplicar técnicas ni herramientas de minería de datos. Además, en los últimos años, el Instituto Tecnológico de Buenos Aires, en conjunto con otras universidades, realiza estudios enfocados en el campo delictivo donde da cuenta de la importancia de aplicar la minería de datos para la exploración y detección de patrones delictivos en Argentina (Valenga et al., 2007), como así también en la inteligencia criminal (Britos et al., 2008).

\section{Metodología}

La metodología aplicada para construir la RSL se basó en el método propuesto por Kitchenham (2004), que consta de las siguientes tres etapas y actividades asociadas con cada una.

Etapa 1. Planificación de la revisión, constó de:

- Definición del objetivo y las preguntas de investigación

- Definición de los términos de búsqueda

- Identificación de las bases de datos y motores de búsqueda por utilizar

- Definición de los criterios de inclusión y exclusión para la RSL

Etapa 2. Desarrollo de la revisión, consistió en lo siguiente:

- Búsqueda en bases de datos científicas y extraer contenidos y datos relevantes

- $\quad$ Selección de estudios primarios

Etapa 3. Reporte de los resultados

- Se analizaron los resultados en función de las preguntas de investigación planteadas en la Etapa 1 de esta indagación.

\section{Resultados}

Los resultados se exponen siguiendo la revisión sistemática de la literatura construida para el objeto de estudio, considerando las tres etapas establecidas en Kitchenham (2004). 
Etapa 1. Planificación de la RSL

\subsection{Definir el objetivo y las preguntas de investigación}

El principal objetivo del documento es presentar una RSL para estudiar e identificar reportes de trabajos y experiencias relacionados con la integración de los procesos de explotación de información con los sistemas de información geográfica, y su utilización en el hallazgo de patrones delictivos. Lo expuesto permitirá conocer el estado actual y sintetizar la literatura existente. Atendiendo a este objetivo, en la tabla 1 se plantearon las preguntas de investigación que guiaron el trabajo.

Tabla 1. Preguntas de investigación formuladas

\begin{tabular}{|c|c|}
\hline Id. Pregunta & Descripción \\
\hline $\mathrm{PI}-1$ & ¿Qué tipo de propuesta se elaboraron para integrar métodos de minería de datos con herramientas GIS? \\
\hline $\mathrm{PI}-2$ & ¿Cómo se ha validado la propuesta de integración? \\
\hline $\mathrm{PI}-3$ & ¿Existe algún artefacto software que automatice la propuesta? \\
\hline $\mathrm{PI}-4$ & ¿Cuáles son los métodos de minería de datos actualmente aplicados al hallazgo de patrones delictivos? \\
\hline PI- 5 & ¿Cuáles son los beneficios y limitaciones de aplicar minería de datos en el análisis delictual? \\
\hline PI- 6 & $\begin{array}{l}\text { ¿Qué herramientas o tecnologías GIS se aplican actualmente para el hallazgo de patrones delictivos y } \\
\text { cuáles son los principales conceptos que están siendo investigados? }\end{array}$ \\
\hline PI- 7 & ¿Cuál es la confiabilidad y el rendimiento de aplicar GIS en el análisis delictivo? \\
\hline
\end{tabular}

\subsection{Definir los términos de búsqueda}

Para formar la cadena de búsqueda se considera una serie de palabras clave y sus palabras relacionadas, como se observa en la tabla 2.

Tabla 2. Definición de la cadena de búsqueda

\begin{tabular}{lc}
\hline \multicolumn{1}{c}{ Palabras clave } & Palabras relacionadas \\
\hline Data Mining & Process information exploitation, \\
& Knowledge Discovery in Databases \\
Geographic Information System & GIS \\
Crime Analysis & criminal analysis, criminal patterns \\
\hline
\end{tabular}

Se utilizaron los operadores lógicos; y se unieron con la conjunción and las palabras clave, y con or las palabras relacionadas. Se obtuvo la siguiente cadena de búsqueda:

(Data mining or Process Information Exploitation or Knowledge Discovery in Databases) and (geographic information system or GIS) and (crime analysis or criminal analysis or criminal patterns). 
1.3. Identificar las bases de datos y motores de búsqueda por utilizar

La ejecución de la búsqueda de los estudios primarios se realiza en el período comprendido entre el 1 de enero de 1990 y el 1 de octubre de 2017, aplicando en los siguientes motores de búsqueda web:

- IEEE Digital Library

- $\quad$ Science Direct

- ACM Digital Library

- Google Scholar

- Servicio de Difusión de la Creación Intelectual (SEDICI), repositorio institucional de la Universidad Nacional de La Plata

\subsection{Definir criterios de inclusión y exclusión para la SLR}

Se consideraron los siguientes criterios de inclusión:

- Artículos relacionados con el tema de interés mediante el análisis del título, el abstract y las palabras clave.

- Además, se analizó como se trataban las palabras clave en el contenido total de cada artículo para decidir si tenía que ser seleccionado en el contexto de la revisión sistemática como estudio relevante (candidato potencial a convertirse en estudio primario).

- Artículos publicados en congresos, workshops y revistas científicas.

- Libros y tesis doctorales o de maestría.

Se consideraron los siguientes criterios de exclusión:

- Artículos publicados en revistas no indexadas.

- Documentos que sean publicaciones de tutoriales.
Etapa 2. Ejecución de la RSL

2.1. Búsqueda en bases de datos científicas y extraer contenidos y datos relevantes (iterando el proceso en varias etapas)

El criterio de ejecución de la revisión sistemática se basó en un modelo iterativo e incremental.

- Iterativo porque la ejecución (búsqueda, extracción de información y visualización de resultados) de la revisión sistemática se hace primero completamente en una fuente de búsqueda, y luego sobre las otras.

- Incremental porque el documento (que es el producto) de la revisión sistemática crece y evoluciona en cada iteración hasta convertirse en el definitivo (el cual contiene los resultados).

\section{2. Selección de estudios primarios}

Para obtener los estudios primarios, se aplicó el siguiente procedimiento a cada fuente de búsqueda elegida o repositorios:

1. Realizar la localización según la cadena de búsqueda en el título (artículos encontrados), teniendo en cuenta las facilidades que proporciona cada fuente para filtrar artículos.

2. En el resultado obtenido (artículos encontrados), se decidió qué artículos incluir y excluir a partir de la lectura del título y del abstract (artículos restantes).

3. Obtener los estudios primarios a partir de la lectura del texto completo (estudios primarios).

4. Distribuir los artículos localizados en cada fuente al aplicar los tres primeros pasos del procedimiento de búsqueda detallados anteriormente. 
En la tabla 3 se observa la selección de los estudios resultado de aplicar la ejecución de la revisión sistemática.

Tabla 3. Distribución de los artículos encontrados por fuente

\begin{tabular}{lcccc}
\hline Fuente de búsqueda & $\begin{array}{c}\text { Artículos } \\
\text { encontrados }\end{array}$ & $\begin{array}{c}\text { Artículos restantes } \\
\text { (después de leer el } \\
\text { título y el abstract) }\end{array}$ & $\begin{array}{c}\text { Estudios primarios } \\
\text { (obtenidos luego de leer el } \\
\text { texto completo) }\end{array}$ & $\begin{array}{c}\text { Porcentaje por } \\
\text { fuente }\end{array}$ \\
\hline IEEE Digital Library & 1.184 & 47 & 6 & 32 \\
Science Direct & 363 & 32 & 1 & 5 \\
ACM Digital Library & 1.089 & 108 & 3 & 16 \\
Google Scholar & 965 & 90 & 4 & 21 \\
SEDICl & 563 & 54 & 5 & 26 \\
Total & 4.164 & 331 & 19 & 100 \\
\hline
\end{tabular}

En el paso 3 se descartaron los artículos aplicando los criterios de inclusión y exclusión y los artículos repetidos, es decir, aquellos encontrados en otra de las fuentes de búsqueda consultadas. Identificados los estudios primarios, se extrajo información de cada una de las fuentes y se ordenaron cronológicamente.

En la tabla 4 se presentan los estudios primarios que constituyen la presente RSL. En la columna 1 se muestra el repositorio que constituyó la fuente de información; las columnas 2 y 3 resumen los autores y títulos de las obras elegidas; en la columna 3, el ámbito de difusión del trabajo; las columnas 4 y 5, el ámbito y período de publicación; finalmente, la última columna indica la relación de la publicación con las preguntas de investigación que aportan a su corroboración.

Tabla 4. Síntesis de la información seleccionada para construir la presente RSL

\begin{tabular}{|c|c|c|c|c|c|}
\hline Fuente & Título & Autores & $\begin{array}{l}\text { Congreso, workshops, } \\
\text { revistas, libros o tesis } \\
\text { doctorales }\end{array}$ & Año & ID-PI \\
\hline IEEE Digital Library & $\begin{array}{l}\text { Association Rules Mining with GIS: } \\
\text { An Application to Taiwan Census } \\
2000\end{array}$ & C. Chang, S. W. Shyue & $\begin{array}{l}2009 \text { Sixth International } \\
\text { Conference on Fuzzy Systems } \\
\text { and Knowledge Discovery }\end{array}$ & 2009 & $\begin{array}{l}\text { PI- 1, } \\
\text { PI- 2, } \\
\text { PI- } 6\end{array}$ \\
\hline IEEE Digital Library & $\begin{array}{l}\text { Research of GIS-based Spatial } \\
\text { Data Mining Model }\end{array}$ & $\begin{array}{l}\text { W. Jinlin, C. Xi, Z. Kefa, } \\
\text { Z. Haibo, W. Wei }\end{array}$ & $\begin{array}{l}2009 \text { Second International } \\
\text { Workshop on Knowledge } \\
\text { Discovery and Data Mining }\end{array}$ & 2009 & $\begin{array}{l}\text { PI- 1, } \\
\text { PI- 2, } \\
\text { PI- } 6\end{array}$ \\
\hline IEEE Digital Library & $\begin{array}{l}\text { Analysis of Crime Factors } \\
\text { Correlation Based on Data Mining } \\
\text { Technology }\end{array}$ & Z. Ying & $\begin{array}{l}2016 \text { International Conference } \\
\text { on Robots \& Intelligent System } \\
\text { (ICRIS) }\end{array}$ & 2016 & $\begin{array}{l}\text { Pl- } 4, \\
\text { Pl- } 5\end{array}$ \\
\hline IEEE Digital Library & $\begin{array}{l}\text { Data Mining and Predictive } \\
\text { Analytics in Public Safety and } \\
\text { Security }\end{array}$ & C. McCue & Magazine IT Professional & 2006 & $\begin{array}{l}\text { Pl- } 4, \\
\text { Pl- } 5\end{array}$ \\
\hline IEEE Digital Library & $\begin{array}{l}\text { Crime Data Mining: A General } \\
\text { Framework and Some Examples }\end{array}$ & $\begin{array}{l}\text { H. Chen, W. Chung, JJ } \\
\text { Xu }\end{array}$ & Computer & 2004 & $\begin{array}{l}\text { PI- } 4, \\
\text { Pl- } 5\end{array}$ \\
\hline
\end{tabular}




\begin{tabular}{|c|c|c|c|c|c|}
\hline IEEE Digital Library & $\begin{array}{l}\text { An Analysis of Data Mining } \\
\text { Applications in Crime Domain }\end{array}$ & P. Thongtae, S. Srisuk & $\begin{array}{l}\text { 8th International Conference } \\
\text { on Computer and Information } \\
\text { Technology }\end{array}$ & 2008 & $\begin{array}{l}\text { Pl- 4, } \\
\text { PI- } 5\end{array}$ \\
\hline Sciene Direct & $\begin{array}{l}\text { Geographic } \\
\text { Knowledge Discovery and Data } \\
\text { Mining }\end{array}$ & R. Laurini & $\begin{array}{l}\text { Geographic Knowledge } \\
\text { Infrastructure Applications } \\
\text { to Territorial Intelligence and } \\
\text { Smart Cities }\end{array}$ & 2017 & $\begin{array}{l}\text { PI- } 1, \\
\text { PI- } 2\end{array}$ \\
\hline ACM Digital Library & $\begin{array}{l}\text { Criminal network analysis and } \\
\text { visualization }\end{array}$ & J. Xu, H. Chen & $\begin{array}{l}\text { Magazine Communication of } \\
\text { the ACM }\end{array}$ & 2005 & $\begin{array}{l}\mathrm{Pl}-4, \\
\mathrm{PI}-5\end{array}$ \\
\hline ACM Digital Library & $\begin{array}{l}\text { Crime Pattern Detection Using } \\
\text { Data Mining }\end{array}$ & S. Varan Nath & $\begin{array}{l}\text { IEEE/WIC/ACM International } \\
\text { Conference on Web } \\
\text { Intelligence and Intelligent } \\
\text { Agent Technology }\end{array}$ & 2006 & $\begin{array}{l}\text { PI- } 4, \\
\text { PI- } 5\end{array}$ \\
\hline ACM Digital Library & $\begin{array}{l}\text { Mining for offender group detection } \\
\text { and story of a police operation }\end{array}$ & $\begin{array}{l}\text { F. Ozgul, J. Bondy and } \\
\text { H. Aksoy }\end{array}$ & $\begin{array}{l}\text { Proceedings of the sixth } \\
\text { Australasian conference on } \\
\text { Data mining and analytics }\end{array}$ & 2007 & $\begin{array}{l}\text { PI- 4, } \\
\text { PI- } 5\end{array}$ \\
\hline Google Scholar & $\begin{array}{l}\text { Crime Modeling and Mapping using } \\
\text { Geospatial Technologies }\end{array}$ & M. Leitner & Editorial Springer & 2013 & $\begin{array}{l}\text { PI- 6, } \\
\text { PI- } 7\end{array}$ \\
\hline Google Scholar & $\begin{array}{l}\text { Geographic Data Mining and } \\
\text { Knowledge Discovery }\end{array}$ & H. J. Miller, J. Han & $\begin{array}{l}\text { Editorial Chapman \& Hall - } \\
\text { Second Edition }\end{array}$ & 2009 & $\begin{array}{l}\text { PI- 1, } \\
\text { PI- } 2\end{array}$ \\
\hline Google Scholar & $\begin{array}{l}\text { Application of GIS in Crime } \\
\text { Analysis: A Gateway to Safe City }\end{array}$ & $\begin{array}{l}\text { R. Gupta, K. Rajitha, S. } \\
\text { Basu, S.K. Mittal }\end{array}$ & $\begin{array}{l}\text { 14th Annual International } \\
\text { Conference and Exhibition } \\
\text { on Geospatial Information } \\
\text { Technology and Applications }\end{array}$ & 2012 & $\begin{array}{l}\text { PI6, } \\
\text { PI- } 7\end{array}$ \\
\hline Google Scholar & Crime Analysis with Crime Mapping & R. Boba Santos & SAGE Publications & 2016 & $\begin{array}{l}\text { PI-6, } \\
\text { PI- } 7\end{array}$ \\
\hline SEDICl & $\begin{array}{l}\text { Aplicación de minería de datos } \\
\text { para la exploración y detección de } \\
\text { patrones delictivos en Argentina }\end{array}$ & $\begin{array}{l}\text { F. Valenga, I. } \\
\text { Perversi, E. Fernández, } \\
\text { H. Merlino, D. } \\
\text { Rodríguez, P. Britos, y } \\
\text { R. García-Martínez. }\end{array}$ & $\begin{array}{l}\text { XIII Congreso Argentino de } \\
\text { Ciencias de la Computación }\end{array}$ & 2007 & $\begin{array}{l}\text { PI- } 4, \\
\text { PI- } 5\end{array}$ \\
\hline SEDICl & $\begin{array}{l}\text { Explotación de información } \\
\text { aplicada a inteligencia criminal en } \\
\text { Argentina }\end{array}$ & $\begin{array}{l}\text { P. Britos, E. Fernández, } \\
\text { H. Merlino, F. Pollo- } \\
\text { Cataneo, D. Rodríguez, } \\
\text { C. Procopio, C Rancan, } \\
\text { R. García-Martínez }\end{array}$ & $\begin{array}{l}\text { XIII Congreso Argentino de } \\
\text { Ciencias de la Computación }\end{array}$ & 2008 & $\begin{array}{l}\text { PI- 4, } \\
\text { PI- } 5\end{array}$ \\
\hline SEDICl & $\begin{array}{l}\text { Explotación de información } \\
\text { geográfica basada en integración } \\
\text { de ambientes de trabajo }\end{array}$ & $\begin{array}{l}\text { R. García-Martínez, H. } \\
\text { Merlino, D. Rodríguez, } \\
\text { S. Martins, E. } \\
\text { Baldizzoni, E. Diez, H. } \\
\text { Amatriain, F. Ribeiro, A. } \\
\text { Segura, P. Santamaría, } \\
\text { F. Mieres, D. Aguirre }\end{array}$ & $\begin{array}{l}X X \text { Congreso Argentino de } \\
\text { Ciencias de la Computación }\end{array}$ & 2014 & $\begin{array}{l}\text { Pl- 1, } \\
\text { Pl- 2, } \\
\text { Pl- 3, } \\
\text { Pl- 4, } \\
\text { Pl- 5, } \\
\text { Pl- 6, } \\
\text { Pl- 7 }\end{array}$ \\
\hline
\end{tabular}




\begin{tabular}{|c|c|c|c|c|c|}
\hline SEDICI & $\begin{array}{l}\text { Identificación y detección de } \\
\text { patrones delictivos basada en } \\
\text { minería de datos }\end{array}$ & $\begin{array}{l}\text { L. Perversi, F. Valenga, } \\
\text { E. Fernández, P. Britos, } \\
\text { R. García-Martínez, }\end{array}$ & $\begin{array}{l}\text { IX Workshop de } \\
\text { Investigadores en Ciencias de } \\
\text { la Computación }\end{array}$ & 2007 & $\begin{array}{l}\text { PI- 4, } \\
\text { PI- } 5\end{array}$ \\
\hline SEDICI & $\begin{array}{l}\text { Identificación de patrones } \\
\text { característicos de la población } \\
\text { carcelera mediante minería de } \\
\text { datos }\end{array}$ & $\begin{array}{l}\text { P. Gutiérrez Rüegg, H. } \\
\text { Merlino, C. Rancan, } \\
\text { C. Procopio, D. } \\
\text { Rodríguez, P. Britos, R. } \\
\text { García-Martínez }\end{array}$ & $\begin{array}{l}X \text { Workshop de Investigadores } \\
\text { en Ciencias de la Computación }\end{array}$ & 2008 & $\begin{array}{l}\text { Pl- 4, } \\
\text { PI- } 5\end{array}$ \\
\hline
\end{tabular}

\section{Etapa 3. Reporte de resultados}

\subsection{Análisis de los resultados}

Los resultados de los estudios primarios se exponen según las preguntas de investigación planteados en la tabla 1.

\subsection{1. ¿Qué tipo de propuesta se elaboraron para integrar} métodos de minería de datos con herramientas GIS? (PI-1)

Se analizaron los 19 estudios primarios para determinar las propuestas de integración de métodos de minería de datos con tecnologías o herramientas GIS. En la tabla 5 se observa la distribución de estos estudios, considerando la propuesta de cada uno. En la categoría Conocimiento se han incluido aquellos artículos que muestran evidencia empírica de la propuesta para integrar la minería de datos con GIS, es decir, la evidencia recogida mediante experimentos o investigación en acción.

Existe un artículo en donde se aplica una extensión de un software GIS para integrar con las técnicas de minería de datos. Este no está disponible para su utilización por el público general.

Bajo la categoría Otras, se consideraron los artículos que proponen métodos no formales: casos de estudios o que no especifican cómo se realizó la integración. Finalmente, la categoría No existe propuesta, hace referencias a aquellos artículos que no proponen ningún método de integración.

Lo expuesto permite inferir la existencia real de un nicho para aportar con un procedimiento que integre el objeto de estudio: es decir, las tecnologías de minería de datos y GIS.

Tabla 5. Estudios primarios, categorías de tipos de propuesta

\begin{tabular}{lcc}
\hline \multicolumn{1}{c}{ Categorías } & Medidas & Porcentuales \\
\hline Conocimiento & 2 & 11 \\
Extensión de software & 1 & 5 \\
Sin propuesta de integración & 14 & 73 \\
Otras & 2 & 11 \\
\hline
\end{tabular}

3.1.2. ¿Cómo se ha validado la propuesta de integración? $(\mathrm{Pl}-2)$

En los estudios primarios, en referencia a la validación de la propuesta de integración, se observa en la tabla 6 que la mayoría de los artículos solo se limitan a mostrar un ejemplo o casos de uso para ilustrar la viabilidad de aquella. Lo expuesto demuestra que es sumamente necesaria la validación de las propuestas para recoger resultados empíricos sobre la efectividad de su uso. 
Tabla 6. Métodos de validación de las propuestas en los estudios primarios

\begin{tabular}{lcc}
\hline Categorías & Medidas & Porcentuales \\
\hline Incluye un caso de estudio & 3 & 17 \\
Incluye un ejemplo & 1 & 5 \\
Solo propuesta & 1 & 5 \\
Carece de propuesta & 14 & 73 \\
\hline
\end{tabular}

3.1.3. ¿Existe algún artefacto software que automatice la propuesta? (PI-3)

Analizando los estudios primarios hallados, se observó que existe un artículo en donde se aplica una extensión de un software GIS para integrar con tecnologías de minería de datos, el cual se desarrolló y formó parte de un proyecto de investigación desarrollado en la Universidad Nacional de Lanús. Sin embargo, actualmente esta aplicación no está disponible para su uso o descarga por el público en general.
3.1.4. ¿Cuáles son los métodos de minería de datos actualmente aplicados al hallazgo de patrones delictivos? (PI-4)

De los artículos primarios elegidos, se analizó que en su mayoría los métodos de minería de datos utilizados para hallar patrones delictivos se basan en el uso de las siguientes técnicas (figura 1). Se distingue que las técnicas de aprendizaje no supervisado para detectar la agrupación de clases son los más elegidos, seguido por las técnicas de aprendizajes supervisados para la clasificación y las técnicas de asociación.

Figura 1. Distribución por técnicas de minería de datos aplicadas al hallazgo de patrones delictivos

\section{Técnicas de minería de datos}

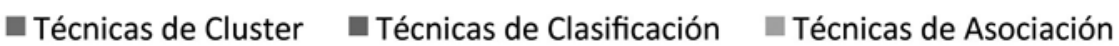

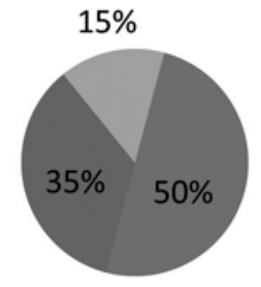

3.1.5. ¿Cuáles son los beneficios y limitaciones de aplicar minería de datos en el análisis delictual? (PI-5)

Se observó, en los estudios primarios, cuáles son los principales beneficios y las limitaciones de aplicar minería de datos al análisis delictual; y son los siguientes:

- Los beneficios del análisis predictivo aplicado a la seguridad y la protección son la identificación temprana y caracterización de una posible amenaza presente.

- Mediante la aplicación de estas técnicas, la prevención es casi siempre menos costosa que la recuperación, particularmente cuando se mide en términos humanos.

- Las técnicas de agrupamiento permiten identificar sospechosos que ejecutan crímenes similares, o para 
distinguir entre grupos pertenecientes a diferentes pandillas.

- Los investigadores pueden aplicar minería de reglas de asociación para descubrir perfiles de los intrusos en una red, con el fin de ayudar a detectar una futura red potencial de ataques.

- La clasificación encuentra propiedades comunes entre diferentes entidades delictivas y las organiza en clases predefinidas.

- Algunas de las limitaciones de usar estas técnicas incluyen que el análisis del patrón del crimen solo puede ayudar al detective, no sustituirlo. Además, la minería de datos es sensible a la calidad de datos de entrada, que pueden ser inexactos. La falta de información también es una problemática; y puede ser propensa (LA MINERÍA) a errores dado el ingreso erróneo de datos.

3.1.6. ¿Qué herramientas o tecnologías GIS se aplican actualmente para el hallazgo de patrones delictivos y cuáles son los principales conceptos que están siendo investigados? (PI-6)

Se determinó en los 19 estudios primarios, las tecnologías GIS actualmente aplicadas para el hallazgo de patrones delictivos. En la Tabla 7 se muestra el número de trabajos que cumple este criterio por herramienta.

Tabla 7. Herramientas GIS utilizadas en el análisis delictual

\begin{tabular}{lcc}
\hline \multicolumn{1}{c}{\begin{tabular}{c} 
Herramientas \\
\multicolumn{1}{c}{ GIS }
\end{tabular}} & $\mathrm{n}^{\circ}$ & Representación \\
& de evidencias & porcentual \\
\hline ArcGIS Desktop & 2 & 11 \\
gvSIG Desktop & 3 & 16 \\
Otros & 1 & 5 \\
No aplican herramientas & 13 & 68 \\
\hline
\end{tabular}

Se investigó el uso del software bajo licencia ArcGIS Desktop comercializado por la empresa ESRI. Este GIS permite ejecutar diversas tareas relacionadas con estadísticas espaciales y con determinados procesos centrados en el análisis del crimen.

Se relevó el uso de un software llamado gvSIG, paquete de software de uso libre para el manejo de información geográfica con precisión cartográfica.

En la categoría Otros, se incluyen aquellos artículos que mencionan el uso de tecnologías GIS, sin especificar el nombre de la herramienta. La categoría No aplica ninguna herramienta, contiene aquellos artículos que no aplican ninguna herramienta GIS.

Según los estudios primarios, los principales conceptos que están siendo investigados de la aplicación de GIS en el análisis de delitos son los siguientes:
- Generación de mapas del delito: que ofrecen una imagen rápida, concreta y fácilmente interpretable de la intensidad con que los hechos delictivos se producen.

- Generación de las zonas calientes: o área geográfica que presenta un nivel de delitos o desorden más elevado que el promedio. Son agrupaciones y conglomerados de delitos que pueden existir a diferentes escalas.

- Identificación de los puntos calientes donde ocurren los delitos.

3.1.7. ¿Cuál es la confiabilidad y el rendimiento de aplicar GIS en el análisis delictivo? (PI-7)

Se observaron, en los estudios primarios, los principales beneficios y las limitaciones de aplicar tecnologías GIS al análisis delictual, entre los que se mencionan los siguientes:

- Los GIS permiten que el personal de las fuerzas de seguridad puedan planificar de forma efectiva las 
respuestas ante emergencias, la determinación de las prioridades en las medidas de mitigación, el análisis de eventos pasados y la predicción de eventos futuros.

- El uso de los GIS en el análisis de delitos facilita el mapeo, la visualización y la observación de puntos calientes del crimen.

- La tecnología GIS ayuda, a los oficiales del crimen, a determinar sitios potenciales de crimen al examinar complejos criterios aparentemente no relacionados y al mostrarlos todos en un mapa.

\section{Conclusiones}

En este artículo se presentó una revisión sistemática de la literatura basada en el método propuesto por Kitchenham. Este permitió recopilar y analizar la literatura existente sobre la integración de procesos de explotación de información con tecnologías GIS y su aplicación para el hallazgo de patrones delictivos.

Se localizaron 19 estudios primarios publicados en el período comprendido entre el 1 de enero de 1990 y el 1 de octubre de 2017. Dichos artículos se analizaron según las preguntas de investigación propuestas en el trabajo. Una reflexión de los hallazgos permite concluir que:

- La aplicación de las tecnologías GIS es, sin lugar a dudas, un tema de suma actualidad para el análisis de patrones delictivos. Dado el estudio de los artículos, se ha podido observar su aplicación y utilidad.

- La utilización de las tecnologías GIS y las técnicas de minería de datos, han demostrado tener un potencial sumamente alto para el análisis de delitos en cualquier región. Las características que aportan para este tipo de análisis son consideradas una de las más importantes, dado que reflejan claridad y conocimiento clave para entender la problemática.

- Existe una mayor tendencia a las propuestas basadas en casos de uso, para lograr entender su aplicación y validarla. También existen artículos que basan la demostración utilizando software GIS bajo una licencia paga.

- Se observa una clara necesidad de validar las propuestas mediante métodos empíricos.

- Es fundamental disponer de algún procedimiento claro y preciso que aporte a la integración de ambas herramientas, dado su amplia utilidad en el área.
El análisis presentado aportará en la definición de un procedimiento que integre ambas tecnologías: los procesos de explotación de la información de la minería de datos y los GIS, y validarlo para determinar los patrones delictivos acontecidos en algún espacio geográfico y tiempo determinado.

\section{Referencias}

El-Aziz, E. M. A., Mesbah, S., \& Mahar, K. (2012). GIS-based de cision support system for criminal tracking. IEEE. (22), 30-31. doi: 10.1109/ICCTA.2012.6523543

Allred, B. J., Daniels, J. J., \& Ehsani, M. R. (2008). Integration of geographic systems (GISS) with agricultural geophysics. En C. J. Merry (Ed.), Handbook of agricultural geophysics. (199-208). New York: CRC Press, Taylor \& Francis Group.

Benet Rodríguez, M., Zafra, S. L., \& Quintero Ortega, S. P. (2015). La revisión sistemática de la literatura científica y la necesidad de visualizar los resultados de las investigaciones. Revista Logos, Ciencia \& Tecnología. Recuperado de https://www.redalyc.org/articulo. oa? $i d=517751487013$

Bernhardsen, T. (2002). Geographic information systems: an introduction. ( $3^{\mathrm{a}}$ ed.). New York: John Wiley \& Sons.

Britos, P. (2008). Procesos de explotación de información basados en sistemas inteligentes. Tesis Doctoral. Universidad Nacional de La Plata, Buenos Aires, ARG.

Britos, P., Fernández, E., Merlino, H., Pollo Cataneo, F., Rodríguez, D., Procopio, C. \& García Martínez, R. (2008). Explotación de información aplicada a inteligencia criminal en Argentina. [Versión de Adobe Acrobat Reader]. Recuperado de http://sedici.unlp. edu.ar/bitstream/handle/10915/21890/Documento_ completo.pdf?sequence $=1$ \&isAllowed $=\mathrm{y}$

Chen, H., Chung, W., Xu, J. J., Wang, G., Qin, Y. \& Chau, M. (2004). Crime data mining: a general framework and some examples. Computer. 37(4), 50-56. doi: 10.1109/ MC.2004.1297301

Fayyad, U., Piatetsky Shapiro, G. \& Smyth, P. (1996). From data mining to knowledge discovery in databases. Al Magazine. 17(3), 39-54.

Ferreira, J. E., Takai, O. K. \& Pu, C. (2005). Integration of business processes with autonomous information systems: a case study in government services. IEEE. (7). doi: 10.1109/ICECT.2005.56 
Frawley, W. J., Piatetsky Shapiro, G. \& Matheus, C. J. (1992). Knowledge discovery in databases: an overview. Al Magazine. 13(5), 58-70.

García Martínez, R., Britos, P., Pesado, P., Bertone, R., Pollo Cattaneo, F., Rodríguez, D. \& Vanrell, J. (2011). Towards an information mining engineering. Software Engineering: Methods, Modeling and Teaching. 83-99.

Gisbert, J. \& Bonfill, X. (2004). ¿Cómo realizar, evaluar y utilizar revisiones sistemáticas y metaanálisis?. Gastroenterología y Hepatología. 27(3), 129-149. doi: 10.1016/S0210-5705(03)79110-9

Hann, I., Hui, K., Lee, S. T. \& Png, I. P. L. (2007). Analyzing online information privacy concerns: an information processing theory approach. IEEE. 210-219.

Kitchenham, B. (2004). Procedures for performing systematic reviews. [Versión de Adobe Acrobat
Reader]. Recuperado de http://www.inf.ufsc.br/ aldo. vw/kitchenham.pdf

Perversi, I., Valenga, F., Fernández, E., Britos, P. V. \& García Martínez, R. (2007). Identificación y detección de patrones delictivos basada en minería de datos. [Versión de Adobe Acrobat Reader]. Recuperado de http://laboratorios.fi.uba.ar/Isi/WICC-07-385389.pdf

Tillekens, A., Le Khac, N. A. \& Thi, T. T. P. (2016). A bespoke forensics GIS tool. IEEE. 1, 1-2. doi: 10.1109/ CSCI.2016.0189

Valenga, F., Perversi, I., Fernández, E., Merlino, H., Rodríguez, D., Britos, P. \& García Martínez, R. (2007). Aplicación de minería de datos para la exploración y detección de patrones delictivos en Argentina. Recuperado de http://sedici.unlp.edu.ar/handle/10915/21783 